\title{
Sustainable pharmacy
}

\section{3.-24. April 2008, Osnabrück, Deutsche Bundesstiftung Umwelt}

\author{
Klaus Kümmerer · Florian Keil • Maximilian Hempel • Hans-Christian Schaefer
}

Erhalten: 26. September 2008/Akzeptiert: 26. September 2008/Online veröffentlicht: 22. Oktober 2008

(C) Springer-Verlag 2008

Arzneimittel sind unentbehrlich für eine hohe Lebensqualität. In den vergangenen Jahren hat sich jedoch gezeigt, dass ihre Anwendung auch eine Kehrseite hat: Arzneimittel sind zumindest in Spuren weit verbreitet in der Umwelt nachweisbar. Sie werden bei der Abwasserbehandlung nur unvollständig entfernt, zum Teil können einzelne Wirkstoffe sogar die Trinkwasseraufbereitung passieren.

Vom 24. bis 25. April 2008 fand in Osnabrück Zentrum für Umweltkommunikation der Deutschen Bundesstiftung Umwelt die erste Internationale Tagung zum Thema „Nachhaltige Pharmazie“ statt. Sie wurde von der Deutschen Bundesstiftung Umwelt, dem Institut für Sozialökologische Forschung und dem Universitätsklinikum Freiburg gemeinsam veranstaltet.

Es bestand unter den Teilnehmern der Tagung Übereinstimmung darin, dass Arzneimittel im Trinkwasser zwar kein akutes Risiko für den Menschen darstellen, dass jedoch insbesondere hinsichtlich möglicher Langzeitfolgen Unsicherheiten bestehen. Arzneimittel sind als Spurenschadstoffe im Wasserkreislauf auf jeden Fall unerwünscht.

In Anbetracht der anerkannten Bedeutung von Arzneimitteln und dem daraus folgenden Faktum, dass sie weder verboten werden können noch sollten, stellt sich die Frage:

\footnotetext{
K. Kümmerer $(\bowtie)$
}

Institut für Umweltmedizin und Krankenhaushygiene am Universitätsklinikum Freiburg,

Breisacher Straße 115 B, 79106 Freiburg, Deutschland

E-Mail: Klaus.Kuemmerer@uniklinik-freiburg.de

F. Keil

Institut für sozial-ökologische Forschung ISOE,

Hamburger Allee 45, 60486 Frankfurt am Main, Deutschland

M. Hempel $\cdot$ H.-C. Schaefer

DBU - Deutsche Bundesstiftung Umwelt,

An der Bornau 2, 49090 Osnabrück, Deutschland
Was sind nachhaltige Strategien, um die genannte Problematik anzugehen?

In der Nachhaltigen Chemie hat sich gezeigt, dass es notwendig ist, die umweltrelevanten Eigenschaften eines chemischen Stoffes entlang allen Lebenswegstationen von der Gewinnung der Rohstoffe über die Verwendung bis zur Entsorgung und dem Verbleib in der Umwelt zu betrachten. Nachhaltige Pharmazie ist eine Version, die aufbauend auf den Erfahrungen der Nachhaltigen Chemie ein entsprechendes Denken auf die Pharmazie überträgt. Eine nachhaltige Pharmazie hat nicht nur die effiziente Wirkung im Blick, sondern betrachtet über die effiziente Nutzung der Ressourcen die Herstellung und die Synthese die Wirkstoffe selbst, die sowohl für die therapeutische Wirksamkeit als auch für den Verbleib in der Umwelt optimiert sind. Eingeschlossen ist darin auch die Frage, wie es gelingen kann, den nachhaltigen Gebrauch von Arzneimitteln zu fördern.

Diese Aspekte wurden bei dieser ersten Konferenz für Nachhaltige Pharmazie (http://www.dbu.de/550artikel 27309_788.html) im internationalen Rahmen angesprochen und diskutiert. Nach der Einführung in das Thema wurden im Rahmen einer Podiumsdiskussion, die ebenfalls international besetzt war, mit Vertretern aus der Industrie, Wasserversorgern und Behörden erste Problemkreise und Handlungsbedarf identifiziert.

Auf der Rohstoffseite werden angesichts der im Vergleich zur chemischen Industrie eher geringen benötigten Mengen an Ausgangsstoffen bisher keine größeren Herausforderungen gesehen. Wenn einzelne Rohstoffe in großer Menge nur aus einer Pflanze gewonnen werden können, kann dadurch der Bestand gefährdet sein. Allerdings sind im Sinne der Nachhaltigkeit ggf. auch Fragen der Patentierung von Naturstoffen zu diskutieren.

Im ersten Vortragsblock wurden relevante Fragen der Produktion von Arzneimitteln, und was diesbezüglich von 
Abb. 1 Zeitskalen der verschiedenen technischen, stofflichen und akteurbezogenen Maßnahmen auf unterschiedlichen Ebenen und unterschiedlichen Zeitskalen

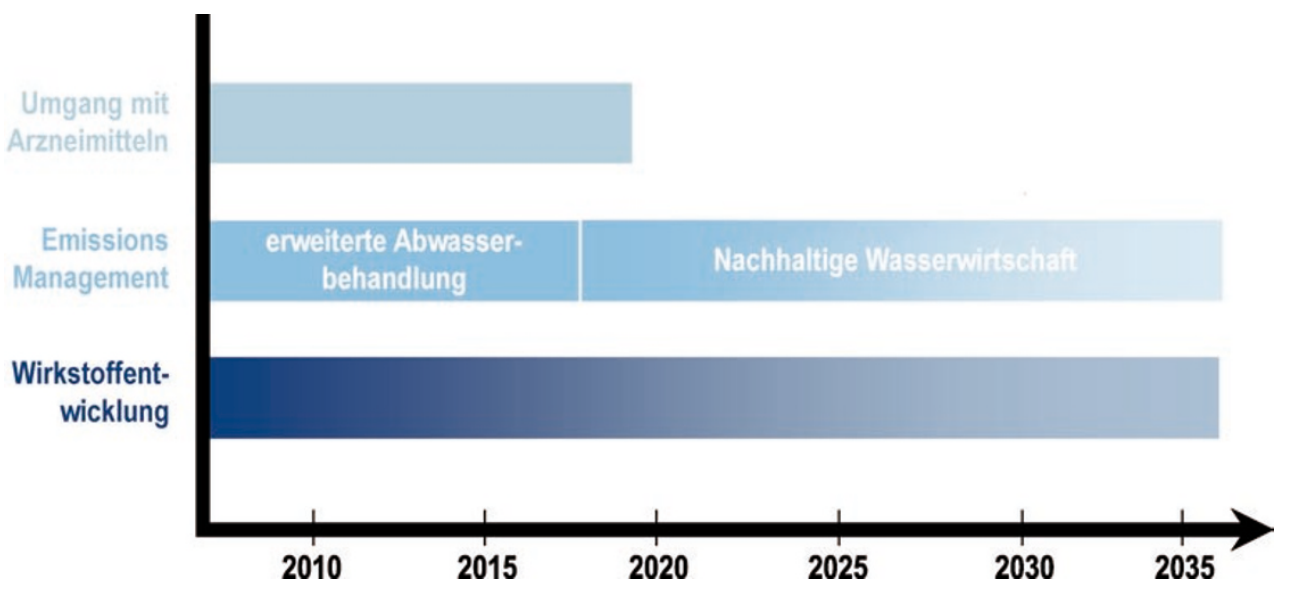

der nachhaltigen Chemie gelernt werden kann, aus Sicht von Hochschulen und Industrie angesprochen.

Im Bereich der Synthese wurden in den letzten Jahren in der pharmazeutischen Industrie enorme Anstrengungen unternommen, den Abfallanfall zu verringern und Synthesen und Prozesse zu optimieren. Dies zahlt sich für die Unternehmen direkt aus. Daher und wegen der Nähe zur Chemie sind in diesem Bereich bisher die größten Fortschritte gemacht worden. Prozessdesign ist ebenfalls in diesem Bereich anzusiedeln. In diesem Feld werden zunehmend nachhaltige Aspekte umgesetzt zur Abfallminimierung, zur Energieeinsparung und auch zur Verbesserung der Arbeitssicherheit.

Im zweiten Vortragsblock ging es darum, wie bei der Entwicklung von Arzneimitteln bereits erwünschte Eigenschaften für die Anwendung aber auch für die Umwelt berücksichtigt werden können. Einerseits sind die Werkzeuge für das gezielte Design und die Optimierung von Wirkstoffen hinsichtlich Wirkungen und unerwünschter Nebenwirkungen weit fortgeschritten. Dabei Umweltaspekte mit einzubeziehen ist aber andererseits beim Top-Management der Firmen bisher kein Thema - wenngleich es auf anderen Ebenen in den Firmen durchaus als beachtenswerter Ansatz eingeschätzt wird. Unter anderem könnte dies daran liegen, dass bisher erfolgreiche Beispiele fehlen und noch kein Wirkstoff als ,grün“ vermarktet wird.

Schwerpunkt eines weiteren Blocks war die Frage, welche Rolle Behörden und Verbraucher bei einem sinnvollen und nachhaltigen Umgang mit Arzneimitteln spielen können. Eine Gesamtstrategie für die Emissionsreduktion wurde diskutiert, die auch die Anwender (Patenten, Ärzte, Apotheker) einbezieht.

Danach ging es darum, wie die Emission von Arzneimitteln aus diffusen Quellen in alternative Konzepte für eine neue urbane Wasserinfrastruktur passt und welche Bedeutung die unterschiedlichen Punktquellen, beispielsweise Krankenhäuser und Altersheime, haben. Nach bisher vorliegenden Daten aus unterschiedlichen Ländern sind Kran- kenhäuser und Altersheime keine bedeutenden Quellen, vielmehr werden die größten Arzneimittelmengen aus den Privathaushalten eingetragen. In Oslo hat sich beispielsweise gezeigt, dass der Eintrag durch eine Pharmafirma die wichtigste Quelle bei einem Wirkstoff darstellte.

Nur $24 \%$ der Weltbevölkerung hat Zugang zu einer Abwasserbehandlung. Hier stellt sich die Frage, welche Möglichkeiten zur Reduzierung der Arzneimittelfracht ins Oberflächenwasser bestehen. Neben angepassten Techniken und dezentralen Lösungen ist nicht nur in diesen Ländern, sondern beispielsweise auch bei uns eine geeignete Information von Patienten, aber auch eine diesbezügliche Ausbildung von medizinischem und pharmazeutischem Personal zum richtigen Umgang und Entsorgung von Arzneimitteln notwendig. Wahrscheinlich ist eine Mischung der verschiedenen technischen, stofflichen und akteursbezogenen Maßnahmen auf unterschiedlichen Ebenen und unterschiedlichen Zeitskalen am ehesten zielführend (Abb. 1).

Der letzte Themenblock beschäftigte sich vor allen Dingen damit, welche Bedeutung die Gesetzgebung und der Markt als Treiber und Hürden für Innovationen spielen. Hier bedarf es der Klärung vieler weiterer Fragen, die im Bereich der Zulassung bis hin zu Fragen aus dem Bereich der Wasserrahmenrichtlinie der EU angesiedelt sind. Die größten Anreize zur Entwicklung neuer Wirkstoffe werden durch Gesetzgebung und ökonomische Randbedingungen gegeben sein. Hier wurden unterschiedliche Ansätze vorgestellt bis dahin, dass Banken künftig in ihr Rating entsprechende Aspekte mit einbeziehen werden. Ein direkter Anreiz für die pharmazeutische Industrie, besser abbaubare Wirkstoffe zu entwickeln, besteht darin, die Laufzeit von Patenten für solche neuen Wirkstoffe um drei bis fünf Jahre zu verlängern. Es ist noch zu klären, ob Nachhaltige Pharmazie Teil eines breiteren Ansatzes innerhalb des generellen Managements von Krankheiten, des Gesundheitssystems und des Verbraucherschutzes sein soll und neue Fragen der Ethik und des Marketing berücksichtigt werden sollen. 
Insgesamt hat die Tagung gezeigt, dass es sich um ein innovatives Thema mit hoher Bedeutung und einem hohen Anspruch handelt. Die Teilnehmer und Veranstalter waren sich darüber einig, dass diese Tagung in etwa zwei Jahren eine Fortsetzung finden sollte. Die Vorträge dieser Tagung sowie weitere Aspekte werden als Buch im Springer Verlag im Jahr 2009 veröffentlicht werden: Sustainable Pharmacy, Springer Verlag.

Link zum Tagungsprogramm: http://www.dbu.de/media/ 041207025458f128.pdf 